\title{
Frailty syndrome in ambulatory patients with COPD
}

\author{
This article was published in the following Dove Press journal: \\ International Journal of COPD \\ 18 April 2017 \\ Number of times this article has been viewed
}

\author{
Panita Limpawattana' \\ Siraphong Putraveephong ${ }^{2}$ \\ Pratchaya Inthasuwan ${ }^{2}$ \\ Watchara Boonsawat ${ }^{3}$ \\ Daris Theerakulpisut ${ }^{4}$ \\ Jarin Chindaprasirt ${ }^{5}$ \\ 'Division of Geriatric Medicine, \\ ${ }^{2}$ Department of Internal \\ Medicine, ${ }^{3}$ Division of Respiratory \\ System, Department of Internal \\ Medicine, ${ }^{4}$ Division of Nuclear \\ Medicine, Department of Radiology, \\ ${ }^{5}$ Division of Oncology Medicine, \\ Department of Internal Medicine, \\ Faculty of Medicine, Khon Kaen \\ University, Khon Kaen, Thailand
}

\begin{abstract}
Frailty is a state of increased risk of unfavorable outcomes when exposed to stressors, and COPD is one of the several chronic illnesses associated with the condition. However, few studies have been conducted regarding the prevalence of COPD and its related factors in Southeast Asia. The objectives of this study were to determine the prevalence of frailty in COPD patients and to identify the associated factors in these populations. A cross-sectional study of COPD patients who attended a COPD clinic was conducted from May 2015 to December 2016. Baseline characteristics were collected, and the diagnosis of frailty was based on the FRAIL (fatigue, resistance, ambulation, illnesses, and loss of weight) scale. Descriptive statistics were used to analyze baseline data. Factors associated with frailty were analyzed using univariate and multivariate regression analyses. The results showed that the prevalence rates of frailty and pre-frailty were $6.6 \%$ (eight out of 121 cases) and $41.3 \%$ (50 out of 121 cases), respectively, among COPD patients. Fatigue was the most common component of the FRAIL scale that was found more frequently in frail patients than in non-frail patients (odds ratio [OR] 91.9). Factors associated with frailty according to multivariate analyses were comorbid cancer (adjusted OR [AOR] 45.8), at least two instances of nonelective admission over the past 12 months (AOR 112.5), high waist circumference (WC) (AOR 1.3), and presence of sarcopenia (AOR 29.5). In conclusion, frailty affected $6.6 \%$ of stable COPD patients. Cancer, two or more instances of nonelective hospitalization over the past 12 months, high WC, and presence of sarcopenia were associated with frailty. Early identification and intervention in high-risk patients is recommended to prevent or delay the adverse outcomes of frailty.
\end{abstract}

Keywords: frailty syndrome, FRAIL scale, chronic lung disease, sarcopenia

\section{Introduction}

Frailty is a part of geriatric syndrome, and has been described as a loss of ability to adapt to stress due to diminished functional reserves. ${ }^{1-3}$ The condition can be considered a pre-disability state. ${ }^{1}$ In frail patients, exposure to stressors can lead to several serious outcomes including procedural complications, institutionalization, fractures, disability, and death. Furthermore, there are adverse consequences associated with other geriatric syndromes such as falls, mild cognitive impairment, delirium, and incontinence. ${ }^{4-7}$ The mechanisms of frailty are related to immune dysfunction, endocrine changes, stress, and energy response systems. ${ }^{1,8}$ Prevalence of frailty in a given population can range from $5 \%$ to $45 \%$ and increases with age..$^{6,7-11}$ In addition, it is more prevalent in women, as well as in African-American and Latino populations. ${ }^{12}$ This figure varies depending on the tool used to define frailty (eg, the physical frailty phenotype or the FRAIL [fatigue, resistance, ambulation, illnesses, and loss of weight] scale) and the population studied. In addition to age-related decline, chronic diseases can also accelerate the rate of deterioration and increase the severity of frailty. ${ }^{9,12}$ The number of chronic diseases in frail older adults has been shown to be about 1.5 times higher than in those not
Correspondence: Panita Limpawattana Division of Geriatric Medicine,

Department of Internal Medicine, Faculty of Medicine, Khon Kaen University, Khon Kaen 40002, Thailand

Tel +6643363664

Fax +664320249I

Email Ipanit@kku.ac.th
International Journal of COPD 2017:12 II93-II98

Dovepress if in 8

http://dx.doi.org/10.21471COPD.S134233 (c) (1) (8) 2017 Limpawattana et al. This work is published and licensed by Dove Medical Press Limited. The full terms of this license are available at https://www.dovepress.com/terms.php (C) NC and incorporate the Creative Commons Attribution - Non Commercial (unported, v3.0) License (http://creativecommons.org/licenses/by-nd/3.0/). By accessing the work you
hereby accept the Terms. Non-commercial uses of the work are permitted without any further permission from Dove Medical Press Limited, provided the work is properly attributed. For permission 
suffering from the condition. ${ }^{12}$ Chronic diseases reported to be associated with frailty are hypertension, chronic kidney disease, osteoarthritis, depression, coronary heart disease, diabetes mellitus, rheumatoid arthritis, stroke, peripheral vascular disease, congestive heart failure, and COPD. ${ }^{1,12}$

COPD and frailty share common risk factors including aging, smoking, endocrine dysfunction, and deregulated inflammation. ${ }^{8}$ In addition to respiratory tract symptoms, COPD patients might suffer from fatigue, anorexia, weight loss, inability to engage in physical activity, muscle weakness, and osteoporosis which are similar to conditions associated with the frailty phenotype. Frailty and COPD, thus, appear to share a common pathophysiology. ${ }^{1,8}$ In previous studies, the prevalence of frailty in COPD patients has varied from $10.2 \%$ to $57 \%$, depending on the screening tools used, ${ }^{6,8-10}$ and has been found to be related to the severity of COPD based on the degree of airflow limitation (Global Initiative for Chronic Obstructive Lung Disease [GOLD] 2007).4,8 Frailty also predicts incompletion of pulmonary rehabilitation and mortality. ${ }^{9}$ Pulmonary rehabilitation, however, has been found to reverse frailty in patients who respond well to the treatment. ${ }^{9}$ Likewise, many factors associated with frailty appear to be preventable or treatable through methods such as implementing an appropriate exercise program and adequate nutritional management. Early recognition of frailty is important, as the introduction of interventions is likely to prevent or delay functional decline, health care cost, hospitalization, disability, and mortality. ${ }^{1,2,7,9}$

There are limited studies regarding frailty in COPD patients, particularly in Southeast Asia where lifestyle and ethnicity differ from those of other regions. Given the variation in prevalence rates of frailty among COPD patients in prior reports, it would be advisable to use a standardized tool that is validated and practical to evaluate frailty. In this regard, the FRAIL scale would be acceptable, as it is feasible and can be generalized to suit various clinical settings. Therefore, the objectives of this study were to determine the prevalence of frailty in COPD patients and to identify the associated factors in these populations using this scale.

\section{Methods}

\section{Subjects}

This was a substudy of "Sarcopenia in COPD: prevalence and associated factors." It was a cross-sectional study that included COPD patients who were 18 years old or over who visited the COPD clinic at Khon Kaen University's Srinagarind Hospital (Thailand) from May 2015 to December 2016. Patients were excluded if they had other active medical conditions (such as COPD exacerbation) within the preceding 4 weeks, were unable to complete the study due to physical limitations (such as being bedridden), had hemiparalysis, were unwilling to participate in this study, or had limitations with regard to undergoing dual-energy X-ray absorptiometry (DXA) or conditions that would affect DXA results.

\section{Definitions COPD}

COPD was diagnosed in patients according to the criteria laid out by the GOLD, which are characterized by persistent airflow limitation, defined as a post-bronchodilator forced expiratory volume in 1 second $\left(\mathrm{FEV}_{1}\right)<70 \%$ of forced vital capacity (FVC). Severity of COPD is classified into three groups: mild ( $\mathrm{FEV}_{1} \geq 80 \%$ normal), moderate $\left(\mathrm{FEV}_{1}\right.$ $50 \%-79 \%$ normal $)$, and severe ( $\mathrm{FEV}_{1} \leq 49 \%$ normal). Under "patients with severe COPD" category, this study included patients with severe COPD ( $\mathrm{FEV}_{1} 30 \%-49 \%$ normal) and very severe $\mathrm{COPD}\left(\mathrm{FEV}_{1}<30 \%\right.$ normal) based on the GOLD staging system. ${ }^{13}$

\section{Frailty syndrome}

Frailty syndrome was defined as a clinical syndrome meeting three or more of the five phenotypic criteria included in the five-item FRAIL scale: fatigue (feeling exhausted most of the time), resistance (limitations climbing 10 flights of stairs), ambulation (inability to walk alone for $>100 \mathrm{~m}$ ), illness (five to 11 of these diseases: hypertension, diabetes mellitus, cancer, chronic lung disease, heart attack, congesting heart failure, angina, asthma, arthritis, stroke, kidney disease), and loss of weight (unintentional weight loss of $>5 \%$ over a past year). ${ }^{2}$ One point was assigned for each component (a total of 0 indicated best and 5 indicated worst). Pre-frail was defined as a clinical state meeting only one or two of the five phenotypic criteria..$^{5,7,11}$

\section{Sarcopenia}

Criteria for sarcopenia diagnosis were based on the definition presented by the Asian Working Groups for Sarcopenia (AWGS), which consists of low muscle mass and low muscle strength and/or poor physical performance. Skeletal muscle mass was estimated using DXA (General Electric [Lunar-Prodigy] model), and appendicular skeletal muscle mass index (ASMI) was calculated as appendicular skeletal mass/height. ${ }^{2}$ Handgrip strength was measured using a handheld dynamometer (GRIP-D [T.K.K.5401] model), and measurement of physical performance was based on a 6-minute walk distance (6-MWD). The following cut-off values were 
used to identify sarcopenia: ASMI of $\leq 7.0 \mathrm{~kg} / \mathrm{m}^{2}$ for men and $\leq 5.4 \mathrm{~kg} / \mathrm{m}^{2}$ for women, handgrip strength of $<26 \mathrm{~kg}$ for men and $<18 \mathrm{~kg}$ for women, or a gait speed of $<0.8 \mathrm{~m} / \mathrm{s}{ }^{14}$

\section{Procedure}

After obtaining written informed consent, baseline patient data were collected by trained clinical researchers. The demographic information consisted of age, sex, educational level, smoking status, severity of COPD assessed using the most recent pulmonary function test report obtained within the past 6 months, underlying diseases and regular medication use assessed by medical record review and asking the patients, history of falls over the past 12 months, self-reported baseline basic and instrumental activities of daily living (ADLs) assessed using the Barthel ADLs index and Chula index, history of nonelective admission over the past 12 months, weight, height, and systolic and diastolic blood pressure at rest. Weight and height were used to calculate the body mass index (BMI) and waist circumference (WC), and systolic and diastolic blood pressures were presented as mean arterial blood pressure. For sarcopenia diagnosis, muscle mass using DXA, handgrip strength using grip dynamometer, and physical performance using the 6-MWD were evaluated in all patients during the same period. Frailty syndrome was diagnosed using the FRAIL scale. ${ }^{14-16}$

\section{Sample size calculation}

Sample size calculation was based on the primary objective of this study, which was to ascertain the estimated prevalence of frailty in COPD patients using the similar criteria. An estimated prevalence of $5 \%-15 \%$ was derived from existing studies. ${ }^{3,6,11} \mathrm{~A}$ formula for estimating a population proportion with specified absolute precision was used to calculate this. It was determined that a sample size of at least 80 participants would be sufficient to achieve the required significance level of 0.05 .

\section{Statistical analysis}

Descriptive statistics for baseline data were presented as percentage, mean, and standard deviation. If the distribution of these data was not normal, then median and interquartile range were used instead. Effects of factors associated with frailty were evaluated using univariate and multivariate regression analyses. In univariate analysis, crude odds ratios (ORs) and 95\% confidence intervals (CIs) were used to consider the strength of association of the factors examined with frailty. Factors with a $P$-value of $<0.20$ were then entered into a multiple logistic regression model. A $P$-value of $<0.05$ was considered to indicate statistically significant differences, and adjusted ORs (AORs) and 95\% CIs were used to determine the strength of association. All data analyses were carried out using STATA version 10.0 (StataCorp, College Station, TX, USA).

Ethical approval was provided by the Khon Kaen University Faculty of Medicine Ethics Committee as instituted by the Declaration of Helsinki (Number HE581060).

\section{Results \\ Prevalence of frailty and pre-frailty in COPD patients}

During the study period, there were 121 COPD patients who were eligible for enrollment. The prevalence of frailty among these patients was $6.6 \%$ (eight out of 121 cases) according to the FRAIL scale, and $41.3 \%$ of patients were found to be in a pre-frail state (50 out of 121 cases). The baseline characteristics of the studied population are shown in Table 1.

\section{Distribution of frailty components in COPD patients}

Frail patients exhibited all components of the FRAIL scale at a greater rate than non-frail patients $(P<0.05)$ with an OR of 91.9 (95\% CI 10.0, 841.9) for fatigue, 39.5 (95\% CI 4.6, 342.0) for resistance, 39.4 (95\% CI 6.8, 227.6) for ambulation, $67.2(95 \%$ CI 5.9, 766.4) for illness, and 9.6 (95\% CI $1.8,50.1)$ for loss of weight as shown in Figure 1.

\section{Factors associated with frailty in COPD}

Following the univariate analysis (Table 1), age, sex, $\mathrm{FEV}_{1} /$ FVC, cancer, use of systemic steroids, nonelective admission over the past 1 year, $\mathrm{WC}$, and sarcopenia were entered into the multiple regression models, as they have been shown to be significant factors $(P<0.2)$ in prior studies. After multicollinearity was checked, cancer, at least two instances of nonelective admission in the past 12 months, high WC, and presence of sarcopenia were found to have a statistically significant association with frailty in this study (Table 2).

\section{Discussion}

In this study, $6.6 \%$ of COPD patients were identified as suffering from frailty. This is slightly lower than the rate of $10 \%-18 \%$ found in previous studies. ${ }^{8,10,12}$ This difference may be attributed to the study setting and assessment tool used, since the criteria to diagnose frailty in COPD patients in the prior reports were based on the physical frailty phenotype. ${ }^{8-10,12}$ However, earlier studies showed that the FRAIL scale could predict physical limitation and mortality 
Table I Baseline characteristics of the studied population and comparison of frail and non-frail subjects using univariate analyses

\begin{tabular}{|c|c|c|c|c|c|}
\hline Variables $(n=|2|)$ & Frail $(n=8,6.6 \%)$ & $\begin{array}{l}\text { Non-frail } \\
(n=\mid 13,93.4 \%)\end{array}$ & Unadjusted OR & $95 \% \mathrm{Cl}$ & $P$-value \\
\hline Age (years), mean (sd) & $73.5(8.9)$ & $69.8(9)$ & 1.1 & $(0.9, I . I)$ & 0.3 \\
\hline Male, n (\%) & $8(100)$ & $104(92)$ & 1 & - & - \\
\hline \multicolumn{6}{|l|}{ Educational level (years), n (\%) } \\
\hline$\leq 6$ & $5(62.5)$ & $54(47.8)$ & 1 & - & - \\
\hline $7-12$ & I (12.5) & $27(23.9)$ & 0.4 & $(0.4,3.6)$ & 0.4 \\
\hline$>12$ & $2(25)$ & $32(28.3)$ & 0.7 & $(0.1,3.7)$ & 0.7 \\
\hline \multicolumn{6}{|l|}{ Smoking status, n (\%) } \\
\hline No history of smoking & $0(0)$ & $7(6.2)$ & 1 & - & - \\
\hline Ex-smoker & $8(100)$ & $96(85)$ & I & - & - \\
\hline Current smoker & $0(0)$ & $10(8.8)$ & I & - & - \\
\hline $\mathrm{FEV}_{1} / \mathrm{FVC}$, mean (sd) & $55.6(11.7)$ & $56.7(12.0)$ & 0.9 & $(0.9,1.1)$ & 0.8 \\
\hline \multicolumn{6}{|l|}{ Comorbid diseases, n (\%) } \\
\hline Diabetes mellitus & $2(25)$ & $10(8.6)$ & 3.4 & $(0.6,19.3)$ & 0.2 \\
\hline Hypertension & $5(62.5)$ & $4 I(36.3)$ & 2.9 & $(0.7,12.9)$ & 0.2 \\
\hline Dyslipidemia & I (I2.8) & $9(8)$ & 1.7 & $(0.2,14.9)$ & 0.7 \\
\hline Chronic arthritis & $0(0)$ & $10(8.9)$ & 1 & - & - \\
\hline Cancer & $2(25)$ & $4(3.5)$ & 9.1 & $(1.4,59.9)$ & 0.02 \\
\hline \multicolumn{6}{|l|}{ Regular medication uses, $\mathrm{n}(\%)$} \\
\hline Inhaled corticosteroid & $8(100)$ & $105(92.9)$ & 1 & - & - \\
\hline Systemic steroid & $I(\mid 2.5)$ & $\mathrm{I}(0.9)$ & 16 & $(0.9,283.6)$ & 0.1 \\
\hline Oral hypoglycemic drugs & $0(0)$ & $7(6.2)$ & 1 & - & - \\
\hline Statin & $2(25)$ & II (9.7) & 3.1 & $(0.6,17.2)$ & 0.2 \\
\hline NSAIDs & $0(0)$ & $\mathrm{I}(0.9)$ & 1 & - & - \\
\hline $\begin{array}{l}\text { History of fall at least two times over } \\
\text { the past I year, } n(\%)\end{array}$ & $0(0)$ & $3(2.7)$ & 1 & - & - \\
\hline Barthel scores, median (min, max) & $20(20,20)$ & $20(9,20)$ & I & - & - \\
\hline Chula iADLs scores, median (min, $\max$ ) & $9(9,9)$ & $9(7,9)$ & I & - & - \\
\hline \multicolumn{6}{|c|}{ Nonelective admission over the past I year, n (\%) } \\
\hline No admission & $4(50)$ & $85(75.2)$ & I & - & - \\
\hline One time & $2(25)$ & $21(18.6)$ & 2 & $(0.3,11.8)$ & 0.4 \\
\hline Two times & $2(25)$ & $7(6.2)$ & 6.1 & $(0.9,39.2)$ & 0.1 \\
\hline BMI $\left(\mathrm{kg} / \mathrm{m}^{2}\right)$, mean $(\mathrm{sd})$ & $24(2.7)$ & $23(4.2)$ & I.I & $(0.9,1.3)$ & 0.5 \\
\hline WC $(\mathrm{cm})$, mean $(\mathrm{sd})$ & $96.4(6.2)$ & $88.1(12.1)$ & I.I & $(0.9, I . I)$ & 0.1 \\
\hline MAP $(\mathrm{mmHg})$, mean (sd) & $97.6(15.8)$ & $95.8(10.3)$ & 1 & $(0.9, I . I)$ & 0.7 \\
\hline Sarcopenia, n (\%) & $4(50)$ & $25(22.2)$ & 3.5 & $(0.8,15.1)$ & 0.1 \\
\hline Osteoporosis, n (\%) & I (I2.5) & 44 (38.9) & 0.2 & $(0,1.9)$ & 0.2 \\
\hline
\end{tabular}

Abbreviations: OR, odds ratio; $\mathrm{Cl}$, confidence interval; sd, standard deviation; FEV , forced expiratory volume in I second; FVC, forced vital capacity; NSAIDs, nonsteroidal anti-inflammatory drugs; Chula iADLs, Chula instrumental activities of daily living; BMI, body mass index; WC, waist circumference; MAP, mean arterial pressure.

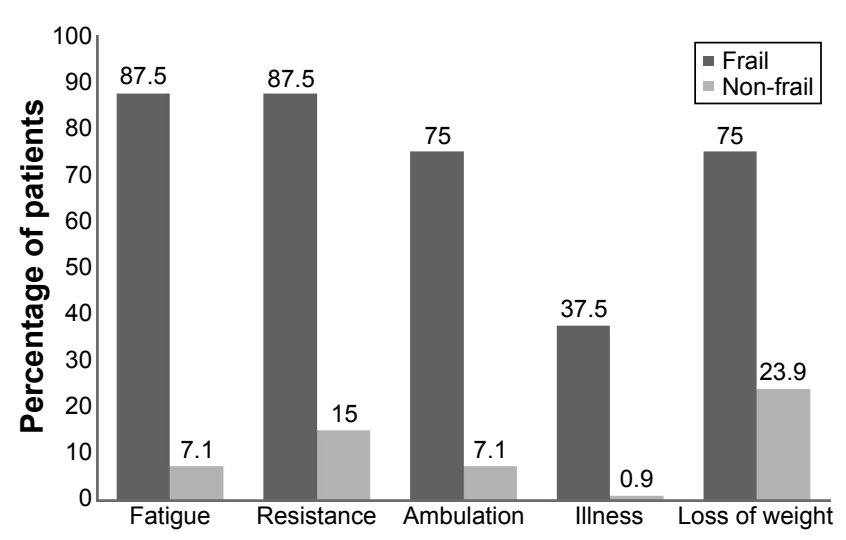

Figure I Distribution of frailty components between frail and non-frail patients by percentage. with accuracy similar to that of the frailty phenotype and multiple deficit model. ${ }^{7}$ A study conducted in the UK found that frailty was prevalent in about one-fourth of all participants, a rate much higher than in other studies. ${ }^{9}$ A possible explanation for this is that the participants in the UK study appeared to have greater severity of airflow limitation and required pulmonary rehabilitation, whereas other studies focused on stable COPD patients in the community or outpatient setting. ${ }^{8,10,12}$ In this study, the fatigue component of the FRAIL scale had the highest magnitude of difference between the frail and non-frail groups. This finding implies that feeling exhausted most of the time is the most sensitive question for frailty screening in COPD patients, though it 
Table 2 Factors associated with frailty using multivariate analysis

\begin{tabular}{|c|c|c|c|}
\hline Variables & Adjusted OR & $95 \% \mathrm{Cl}$ & $P$-value \\
\hline Age & 1.1 & $(0.9,1.3)$ & 0.4 \\
\hline Male & I & - & - \\
\hline$\% \mathrm{FEV}_{1}$ & 1.0 & $(0.9,1.03)$ & 0.3 \\
\hline Cancer & 45.8 & $(2.0,1,050.7)$ & $0.02 *$ \\
\hline $\begin{array}{l}\text { Use of systemic } \\
\text { steroid }\end{array}$ & 225.3 & $(0.5,1,08086.5)$ & 0.1 \\
\hline \multicolumn{4}{|c|}{ Nonelective admission over the past I year } \\
\hline $\begin{array}{l}\text { No } \\
\text { admission }\end{array}$ & 1 & - & - \\
\hline One time & 0.2 & $(0.01,7.7)$ & 0.4 \\
\hline Two times & 112.5 & $(2.0,6,331.4)$ & $0.02 *$ \\
\hline WC & 1.3 & $(I . I, I .6)$ & $0.01 *$ \\
\hline Sarcopenia & 29.5 & $(1.7,521.0)$ & $0.02 *$ \\
\hline
\end{tabular}

Note: $* P$-value $<0.05$

Abbreviations: $\mathrm{OR}$, odds ratio; $\mathrm{Cl}$, confidence interval; $\mathrm{FEV}_{1}$, force expiratory volume in I second; WC, waist circumference.

is subjective and nonspecific. We found that about $40 \%$ of patients were non-frail, which is similar to the findings in former reports of about $18 \%-52 \%{ }^{7,10}$

Cancer, nonelective admission over the past 12 months, WC, and presence of sarcopenia were the factors associated with frailty in this study. The development of frailty among cancer survivors has been documented in earlier studies, which propose that a possible pathophysiology could be iatrogenic damage and residual chronic conditions as the result of cancer and biological aging, leading to clinical expression of frailty. ${ }^{17,18}$ This study found that two or more nonelective admissions over the past 12 months increased the risk of developing frailty. This was the result of both normal changes associated with aging, such as reduced muscle strength, aerobic capacity, and bone mineral density, and the effects of hospitalization such as immobilization and increased bone loss. These factors could lead to deconditioning, dependency, and ultimately, frailty. ${ }^{19}$ Thus, the more times the patients are admitted, the greater their risk of becoming frail. This study found that higher WC escalated the risk of frailty. In general, frailty is thought of mainly as a wasting disorder. Conversely, WC, which reflects central obesity, was also related to this condition, supporting earlier findings that excessive adiposity could lead to physical frailty. This is particularly true when it occurs with reduced muscle mass and/or muscle strength or physical performance, which can be defined as sarcopenia. ${ }^{20,21}$ Importantly, reduced muscle mass might be disregarded in obese patients, until there is distinct functional loss of muscle strength, the so-called sarcopenic obesity. ${ }^{21}$ This study found sarcopenia to have a positive relationship with frailty. This confirms the evidence that sarcopenia has been considered physical frailty, as they both share common pathophysiology and clinical presentations including the effect on inspiratory and expiratory respiratory muscles. . $^{11,14,16,22,23}$

According to several reports, frailty increases with age. ${ }^{2,3,9,10}$ This association was not found in this study, however, probably due to the small sample size. This study also could not demonstrate the link between BMI and frailty in COPD patients. The reason might be that BMI was not a good predictor of adverse outcomes in COPD patients, as numerous studies have shown that BMI is not associated with staging of the disease. This is because the increased extra fat stores result in more severe COPD. Consequently, BMI remained unchanged. ${ }^{24-26}$ The severity of COPD was not associated with frailty in this study, which is similar to the results found in another study conducted in the same setting. ${ }^{10}$ This might be explained by the study design. It included stable COPD patients in an outpatient setting, while studies that showed an association between frailty and the degree of airflow limitation included more severe patients. ${ }^{8}$

As frailty and pre-frailty syndrome were common in COPD patients, and given that clinical outcomes of patients with frailty could be reversed using multidisciplinary intervention (especially at the pre-frailty stage), ${ }^{27}$ early recognition of this syndrome among COPD patients is critical. Examining for risk factors, such as comorbid cancer, a history of hospitalization, over-/undernutrition, or sarcopenia (using handgrip strength and gait speed for screening according to the AWGS recommendation), would be beneficial in order for early intervention, such as comprehensive geriatric assessment, physical therapy referral, and nutritional intervention, to take place..$^{2,3,10}$ This could potentially prevent or delay the progression of frailty.

\section{Limitations}

This study has some limitations. First, certain patients who were likely to be frail but had physical limitations, such as hemiparalysis or being bedridden, were excluded from our study. Second, the patients who were included were ambulatory patients at an outpatient clinic in a tertiary care hospital. Thus, the results might not be generalizable to other settings. Third, it was a cross-sectional study, and the FRAIL scale questionnaires are self-reported, so misclassification bias could have occurred.

\section{Conclusion}

Overall, the prevalence of frailty in COPD patients was 6.6\%, and fatigue was the major component of frailty in these populations as assessed using the FRAIL scale. This study also showed that comorbid cancer, sarcopenia, higher WC, and 
at least two instances of nonelective admission over the past year were associated with frailty syndrome. Therefore, frail COPD patients might benefit from intervention based on these associated factors. Likewise, prevention of sarcopenia and other related factors may have a role in reducing the risk of frailty and mortality in COPD. These issues should be taken into consideration in further clinical trials.

\section{Acknowledgments}

The authors would like to acknowledge Mr Dylan Southard, Research Affairs, Faculty of Medicine, Khon Kaen University, Thailand, for editing the manuscript. This study was funded by the Neuroscience Research and Development Group, Khon Kaen University, Thailand, under grant number 001/2558, the Sleep Apnea Research Group, Khon Kaen University Thailand, and the Thailand Research Fund (number IRG 5780016).

\section{Disclosure}

The authors report no conflicts of interest in this work.

\section{References}

1. Angulo J, El Assar M, Rodríguez-Mañas L. Frailty and sarcopenia as the basis for the phenotypic manifestation of chronic diseases in older adults. Mol Aspects Med. 2016;50:1-32.

2. Espinoza S, Walston JD. Frailty in older adults: insights and interventions. Cleve Clin J Med. 2005;72(12):1105-1112.

3. Morley JE, Vellas B, van Kan GA, et al. Frailty consensus: a call to action. J Am Med Dir Assoc. 2013;14(6):392-397.

4. Uchmanowicz I, Jankowska-Polanska B, Chabowski M, Uchmanowicz B, Fal AM. The influence of frailty syndrome on acceptance of illness in elderly patients with chronic obstructive pulmonary disease. Int J Chron Obstruct Pulmon Dis. 2016;11:2401-2407.

5. Díaz de León González E, Gutiérrez Hermosillo H, Martinez Beltran JA, et al. Validation of the FRAIL scale in Mexican elderly: results from the Mexican Health and Aging Study. Aging Clin Exp Res. 2016; 28(5):901-908.

6. Park SK, Richardson CR, Holleman RG, Larson JL. Frailty in people with COPD, using the National Health and Nutrition Evaluation Survey dataset (2003-2006). Heart Lung. 2013;42(3):163-170.

7. Woo J, Yu R, Wong M, Yeung F, Wong M, Lum C. Frailty screening in the community using the FRAIL scale. J Am Med Dir Assoc. 2015;16(5): 412-419.

8. Lahousse L, Ziere G, Verlinden VJ, et al. Risk of frailty in elderly with COPD: a population-based study. J Gerontol A Biol Sci Med Sci. 2016; 71(5):689-695.
9. Maddocks M, Kon SS, Canavan JL, et al. Physical frailty and pulmonary rehabilitation in COPD: a prospective cohort study. Thorax. 2016; 71(11):988-995.

10. Mittal N, Raj R, Islam EA, Nugent K. The frequency of frailty in ambulatory patients with chronic lung diseases. J Prim Care Community Health. 2016;7(1):10-15.

11. Milte R, Crotty M. Musculoskeletal health, frailty and functional decline. Best Pract Res Clin Rheumatol. 2014;28(3):395-410.

12. Weiss CO. Frailty and chronic diseases in older adults. Clin Geriatr Med. 2011;27(1):39-52.

13. Vestbo J, Hurd SS, Agustí AG, et al. Global strategy for the diagnosis, management, and prevention of chronic obstructive pulmonary disease: GOLD executive summary. Am J Respir Crit Care Med. 2013; 187(4):347-365.

14. Chen LK, Liu LK, Woo J, et al. Sarcopenia in Asia: consensus report of the Asian Working Group for Sarcopenia. J Am Med Dir Assoc. 2014;15(2):95-101.

15. Jitapunkul S, Kamolratanakul P, Ebrahim S. The meaning of activities of daily living in a Thai elderly population: development of a new index. Age Ageing. 1994;23(2):97-101.

16. Limpawattana P, Kotruchin P, Pongchaiyakul C. Sarcopenia in Asia. Osteoporos Sarcopenia. 2015;1(2):92-97.

17. Baijal P, Periyakoil V. Understanding frailty in cancer patients. Cancer J. 2014;20(5):358-366.

18. Pérez-Zepeda MU, Cárdenas-Cárdenas E, Cesari M, Navarrete-Reyes AP, Gutiérrez-Robledo LM. Cancer and frailty in older adults: a nested casecontrol study of the Mexican Health and Aging Study. J Cancer Surviv. 2016;10(4):736-742.

19. Creditor MC. Hazards of hospitalization of the elderly. Ann Intern Med. 1993;118(3):219-223.

20. Porter Starr KN, McDonald SR, Bales CW. Obesity and physical frailty in older adults: a scoping review of lifestyle intervention trials. $J \mathrm{Am}$ Med Dir Assoc. 2014;15(4):240-250.

21. Shah K, Villareal DT. Preventing frailty in obese older adults. J Frailty Aging. 2012;1(2):47-49.

22. Kim TN, Choi KM. Sarcopenia: definition, epidemiology, and pathophysiology. J Bone Metab. 2013;20(1):1-10.

23. Kim TN, Yang SJ, Yoo HJ, et al. Prevalence of sarcopenia and sarcopenic obesity in Korean adults: the Korean sarcopenic obesity study. Int $J$ Obes (Lond). 2009;33(8):885-892.

24. Pothirat C, Chaiwong W, Phetsuk N, et al. The relationship between body composition and clinical parameters in chronic obstructive pulmonary disease. J Med Assoc Thai. 2016;99(4):386-393.

25. Wagner PD. Possible mechanisms underlying the development of cachexia in COPD. Eur Respir J. 2008;31(3):492-501.

26. Ischaki E, Papatheodorou G, Gaki E, Papa I, Koulouris N, Loukides S. Body mass and fat-free mass indices in COPD: relation with variables expressing disease severity. Chest. 2007;132(1):164-169.

27. Acosta-Benito MA, Sevilla-Machuca I. Using prefrailty to detect early disability. J Family Community Med. 2016;23(3):140-144.
International Journal of COPD

\section{Publish your work in this journal}

The International Journal of COPD is an international, peer-reviewed journal of therapeutics and pharmacology focusing on concise rapid reporting of clinical studies and reviews in COPD. Special focus is given to the pathophysiological processes underlying the disease, intervention programs, patient focused education, and self management protocols.

\section{Dovepress}

This journal is indexed on PubMed Central, MedLine and CAS. The manuscript management system is completely online and includes a very quick and fair peer-review system, which is all easy to use. Visit $\mathrm{http}: / / \mathrm{www}$.dovepress.com/testimonials.php to read real quotes from published authors. 